Experiments in Fluids manuscript No.

(will be inserted by the editor)

\title{
Dynamic Stall Development
}

\author{
Karen Mulleners • Markus Raffel
}

Received: date / Accepted: date

\begin{abstract}
Dynamic stall on an oscillating airfoil was investigated by a combination of surface pressure measurements and time-resolved particle image velocimetry. Following up on previous work on the onset of dynamic stall (Mulleners and Raffel 2012), we combined time-resolved imaging with extensive coherent structure analysis to study various aspects of stall development. The formation of the primary dynamic stall vortex was identified as the growth of a recirculation region and the ensuing instability of the associated shear layer. The stall development can be subdivided into two stages of primary and secondary instability with the latter being the effective vortex formation stage. The characteristic time scales associated with the primary instability stage revealed an overall decrease of dynamic stall delay with increasing effective unsteadiness of the pitching airfoil. The vortex formation stage was found to be largely unaffected by variations of the airfoil's dynamics.
\end{abstract}

\section{List of Symbols}

$c$

$C_{1}$

$C_{\mathrm{p}}$

$C_{\mathrm{p}, \min }$

$f_{\text {osc }}$

$k$

$M a$
$N$

$R e$

$t_{\mathrm{ss}}$

$t^{*}$

$t_{\mathrm{ds}}$

$T$

$\mathbf{u}=(u, v, w)$

$U_{\infty}$

$\mathbf{x}=(x, y, z)$

$\mathbf{x}_{\mathrm{vi}}=\left(x_{\mathrm{vi}}, 0, z_{\mathrm{vi}}\right)$

$\mathbf{x}_{\mathrm{w}}=\left(x_{\mathrm{w}}, y_{\mathrm{w}}, z_{\mathrm{w}}\right)$

$\alpha$

$\alpha_{0}$

$\alpha_{1}$

$\alpha^{*}$

$\alpha_{\mathrm{ds}}$

$\alpha_{\mathrm{ss}}$

$\dot{\alpha}_{\mathrm{ss}}$

$\Gamma$

$\nu$

$\Delta t_{1}$

$\Delta t_{2}$

$\Delta z$

$\omega$

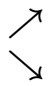

number of clockwise rotating

vortices

Reynolds number

passage of the static stall angle

during upstroke

onset of shear layer roll-up

dynamic stall onset

oscillation period

local velocity

free steam velocity

spatial coordinates

position of the vortex $v_{\mathrm{i}}$

airfoil coordinates

angle of attack

mean incidence

oscillation amplitude

angle of attack at the onset of shear

layer roll-up

dynamic stall angle of attack

static stall angle of attack

instantaneous effective unsteadiness

scalar function

kinematic viscosity

stall delay attributed to the primary

instability stage

stall delay attributed to the vortex

formation stage

average shear layer displacement

vorticity

during upstroke

during downstroke

German Aerospace Center (DLR),

Bunsenstr. 10, 37073 Göttingen, Germany

Present address: of K. Mulleners

Institute of Turbomachinery and Fluid Dynamics,

Leibniz Universität Hannover, Germany

E-mail: mulleners@tfd.uni-hannover.de 

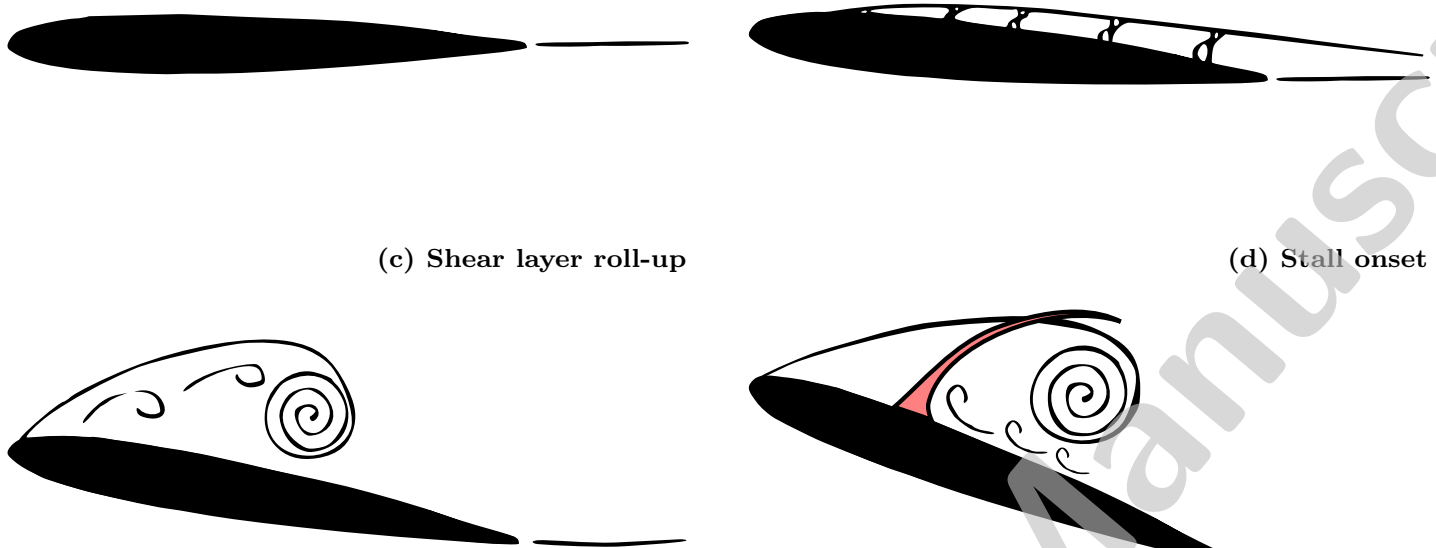

(e) Full stall

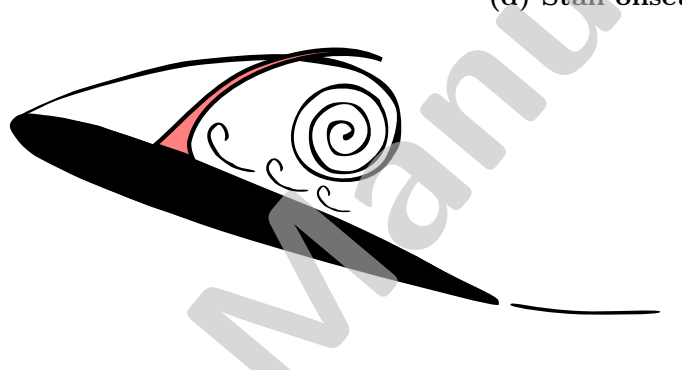

(d) Stall onset

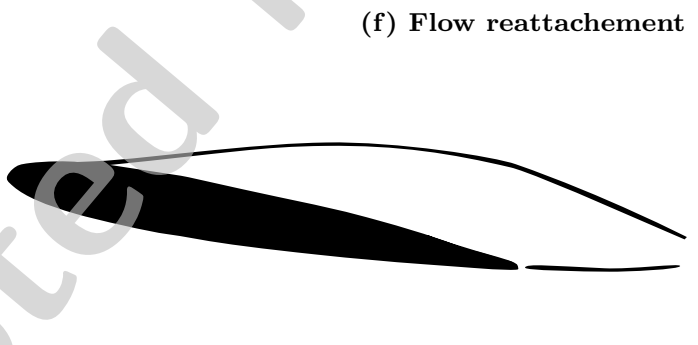

Fig. 1 Schematic representation of the different stages of the dynamic stall process (modified from Doligalski and Smith (1994) and Carr et al (1977)).

\section{Introduction}

Dynamic stall (DS) occurs on helicopter or wind turbine rotor blades in response to an unsteady change of the angle of attack beyond the static stall angle. It is characterised by the formation of a large-scale dynamic stall vortex and a delayed onset of massive flow separation with respect to static stall (McAlister et al 1978; McCroskey 1981; Carr 1988).

Highly unsteady aerodynamic loads during dynamic stall decrease the aerodynamic efficiency while increasing structural forces and bending moments. Since DS is the key to agility, manoeuvrability, and structural stability of rotary wings, improving dynamic stall modelling and prediction capabilities is highly desirable. For this purpose accurate knowledge of the unsteady flow field and associated vortex dynamics during dynamic stall development is essential.

Based on a combination of time-resolved velocity field information and extensive coherent structure analysis, the development of the unsteady flow over an os- cillating airfoil within a single dynamic stall life cycle was previously classified into five successive stages: the attached flow stage; the stall development stage; stall onset; the stalled stage; and flow reattachment (Mulleners and Raffel 2012). Due to the unsteady nature of the process, time-resolved measurement methods are required to analyse the chronology and causality of the prominent stall events (figure 1) (Carr et al 1977; Shih et al 1992).

The stall development stage is the part of the DS life cycle between static and dynamic stall onset covering the unsteady separation process. The flow separation is initiated by an adverse pressure gradient and involves the formation and detachment of a shear layer from the airfoil's surface (Reynolds and Carr 1985; Degani et al 1998). With increasing angle of attack beyond static stall, an adverse pressure gradient builds up and leads to the formation of a prominent recirculation region that grows normal to the surface (figure 1(b)). At the interface between regions of reverse and free stream flow, a shear layer develops that controls the succes- 
sive stall development (Ho 1986). The primary instability of the shear layer generates small-scale vortices that interact only weakly at first. Subsequently, viscous interactions increase leading to the roll-up of the shear layer into a large-scale DS vortex (figure 1(c)). The primary stall vortex subsequently detaches due to vortex induced separation signalising dynamic stall onset (figure 1(d)) (Obabko and Cassel 2002). The onset of dynamic stall can be readily specified directly from the velocity field based on a characteristic mode of the proper orthogonal decomposition (POD) of the velocity field (Mulleners and Raffel 2012).

Following up on that work, this paper focusses on the identification and characterisation of the physical mechanisms and parameters controlling initiation, growth, and subsequent detachment of the DS vortex. In order to sustain and further improve dynamic stall modelling, special emphasis is placed on the role and behaviour of the shear layer and the associated time scales. A combination of time-resolved velocity field information with different coherent structure detection methods serves to fulfil the above objectives.

\section{Materials and Methods}

Unsteady surface pressure and time-resolved particle image velocity (TR-PIV) measurements were conducted in the central cross sectional plane of a pitching OA209 airfoil (figure 2). The velocity field was measured with a spatial resolution of $1.2 \mathrm{~mm}$ at an acquisition rate of $1500 \mathrm{~Hz}$ ( $6 \mathrm{kHz}$ for the pressure distribution). The two-dimensional airfoil model with an OA209 profile of chord length $c=0.3 \mathrm{~m}$ was placed in a uniform flow at free stream Reynolds number $R e=\frac{U_{\infty} c}{\nu}=9.2 \times 10^{5}$ with $U_{\infty}$ the free stream velocity and $\nu$ the kinematic viscosity (Mach number $M a=0.14$ ). The airfoil was subjected to a sinusoidal oscillation about its quarter chord axis with mean incidence $\alpha_{0}$, amplitude $\alpha_{1}$, and reduced frequency $k$. The latter is defined as $k=\pi f_{\text {osc }} c / U$ with $f_{\text {osc }}$ the pitching frequency. Mean incidence, amplitude and reduced frequency were varied to attain different stall cases.

The time-resolved PIV data was evaluated according to standard procedures (Raffel et al 2007) using the multi grid algorithm with image deformation and a final interrogation window size of $32 \mathrm{px} \times 32 \mathrm{px}$ and an overlap of approximately $80 \%$. This yields a grid spacing or physical resolution of $6 \mathrm{px}$ or $1.2 \mathrm{~mm}=0.004 \mathrm{c}$.

The detection probability of a valid displacement in the PIV data was higher than $90 \%$. The remaining spurious vectors were detected and replaced using the automated post-processing algorithm of Garcia (2010).

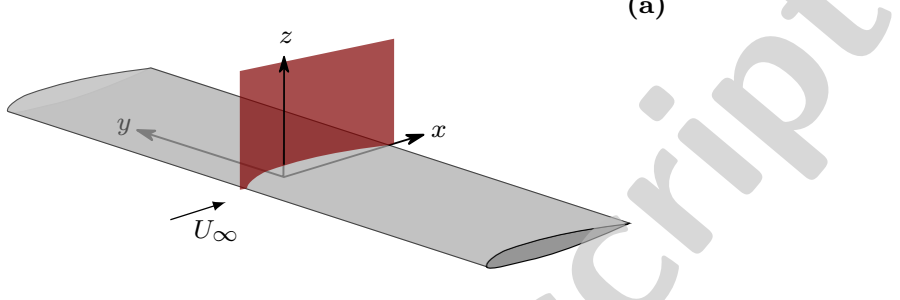

(b)

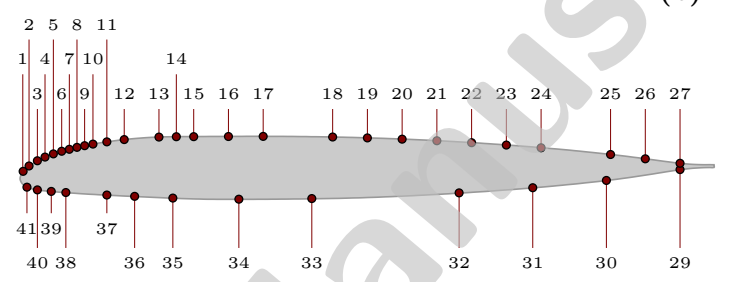

Fig. 2 Position of the PIV field of view (a) and location of the unsteady pressure sensors in the mid-span section of the OA209 airfoil profile (b).

The inaccuracy in determining the valid displacements is given by the single displacement error $\epsilon_{\mathrm{x}}$, which is decomposed into the random error or measurement uncertainty $\epsilon_{\mathrm{rms}}$ and the bias error $\epsilon_{\text {bias }}$. The random error refers to measurement uncertainties induced and affected by the particle image diameter, the size of the interrogation window size, and the particular flow conditions. The interrogation windows size has an important influence on the measurement uncertainty which increases with decreasing interrogation window size Raffel et al (2007). For a good compromise between a high spatial resolution and a low random error, the data was evaluated with a final window size of $32 \mathrm{px} \times 32 \mathrm{px}$. Based on careful analysis of the results, the random error was estimated smaller than $0.05 \mathrm{px}$ for observation areas containing uniform flow and $0.1 \mathrm{px}$ for observation areas where strong velocity gradients are present. The bias error is mainly determined by peak-locking, a phenomenon describing the tendency of the displacements to be biased towards integer pixel values. Histograms of subpixel displacement showed that peak locking was successfully avoided and the remaining bias error was assumed to be significantly less than the random noise error, i.e. $\epsilon_{\text {bias }}<0.05 \mathrm{px}$.

The total error for the velocity measurement is now determined by dividing the single displacement error by the pulse-separation time $\Delta t$ and the magnification factor of the camera recording $M$ according to $\epsilon_{\mathrm{vel}}=\frac{\epsilon_{\mathrm{x}}}{\Delta t M}$. The measurement error is often made dimensionless by the mean free flow velocity yielding a relative error $\epsilon_{\mathrm{rel}}=\epsilon_{\mathrm{v}, \text { in }} / U_{\infty} \leq 0.02$. Due to the recording geometry and random character of the measurement uncertainty, the measurement error for the out-of-plane 
velocity component was estimated to be a factor of 2 higher than for the in-plane components.

Prior to analysis, the velocity fields were rotated into the airfoil reference system with the $x$-axis along the chord, the $y$-axis along the span and the $z$-axis upward perpendicular to the chord, while the origin coincides with the rotation axis, i. e. the airfoil's quarter chord axis, at model mid-span.

The characteristic flow features were identified and analysed using an Eulerian and a Lagrangian procedure. The Eulerian method utilises the dimensionless scalar function $\Gamma$ introduced by Michard et al (1997) to locate the axis of individual vortices and to determine their sense of rotation. The Lagrangian approach adopted was the finite-time Lyapunov exponent (FTLE) method (Haller and Yuan 2000). Particle trajectories were integrated forward and backward in time, yielding positive and negative finite-time Lyapunov exponent fields (pFTLE and nFTLE fields). The ridges in the FTLE fields indicate the boundaries of Lagrangian coherent structures (LCS) (Shadden et al 2005). The FTLE fields were calculated based on the in-plane velocity field with the software package ManGen created by Lekien and Coulliette (Lekien et al 2005).

\section{Results and Discussion}

Following up on the specification of the dynamic stall onset (Mulleners and Raffel 2012), we focus here on the identification and characterisation of the individual physical mechanisms and relevant time scales of the flow development prior to stall onset. To repeat the overall flow development during a single dynamic stall life cycle, instantaneous velocity fields and surface pressure distributions for the harmonic oscillation specified by $\alpha_{0}=20^{\circ}, \alpha_{1}=8^{\circ}$ and $k=0.10$ are depicted in figure 3 for the different stages of the dynamic stall process summarised in figure 1 . The presence and location of clockwise and counterclockwise rotating vortices, as determined by the Eulerian detection algorithm, help to identify the characteristic features of the dynamic stall life cycle.

The first noticeable event that occurs during the upstroke motion is the development of a shear layer at the interface between recirculating flow near the upper rear surface of the airfoil and the free stream flow. The shear layer contains only positive vorticity which is clumped into individual shear layer vortices (figure 3(b)). With increasing angle of attack, the region of flow reversal grows. This is accompanied by an increase in the pressure coefficient at the surface underneath the recirculating flow. The leading edge suction peak continues to grow with increasing angle of attack until the shear

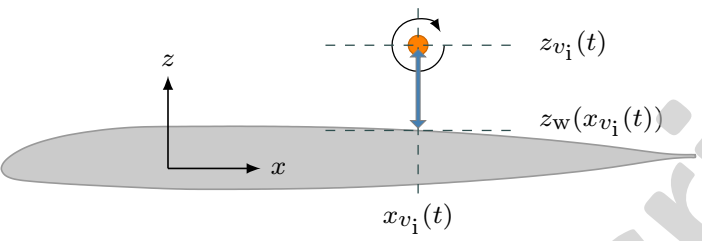

Fig. 4 Schematic representation of the computation of $\Delta z$.

layer roll ups into a large scale DS vortex (figure 3(c)). For a short period of time, the DS vortex grows in size while remaining in place until it has grown strong enough to separate as a result of a vortex induced separation process (figure $3(\mathrm{~d})$ ). The presence of the largescale structure can be recognised by a plateau in the surface pressure distribution between approximately $x / c=$ -0.1 and $x / c=0.2$ on the suction side. This scenario of vortex development, where the shear layer rolls up into a large scale coherent structure which grows locally and temporally until separating is generally referred to as wake mode (Gharib and Roshko 1987; Hudy et al 2007). It differs from the shear layer mode where the shear layer continuously rolls up into large scale structures that grow spatially, i.e. while travelling downstream.

The detachment of the DS vortex marks stall onset and the beginning of the stalled stage which is characterised by massive flow separation (figure $3(\mathrm{e})$ ). The stalled stage lasts for most of the rest of the cycle until, near the end of the downstroke, the flow reattaches from the leading edge towards the trailing edge (figure 3(f)).

\subsection{Shear Layer Displacement}

The prominent feature that plays an important role prior to stall onset is the shear layer. The shear layer limits the recirculation region and it is identified by the small scale shear layer vortices. To quantify and analyse the growth of the recirculation region a new quantity $\Delta z$ is introduced to represent the average chord-normal distance of the shear layer from the airfoil's upper surface. Considering the shear layer as a vortex sheet containing individual small-scale vortices resulting from the primary instability, $\Delta z$ is calculated as the average chord-normal height of all clockwise rotating vortical structures in individual experimental snapshots of the velocity field or

$$
\Delta z(t)=\frac{1}{N(t)} \sum_{i=1}^{N(t)}\left|z_{v_{\mathrm{i}}}(t)-z_{\mathrm{w}}\left(x_{v_{\mathrm{i}}}(t)\right)\right|
$$

Here, $N(t)$ is the total number of clockwise rotating vortices at time $t$ according to the Eulerian vortex detection algorithm and $\left|z_{v_{\mathrm{i}}}(t)-z_{\mathrm{w}}\left(x_{v_{\mathrm{i}}}(t)\right)\right|$ is the chord- 

$-0.04$
$-0.03$
$-0.02$
$-0.01$
0
0.01
0.02
0.03
0.04

$\omega c / U_{\infty}$
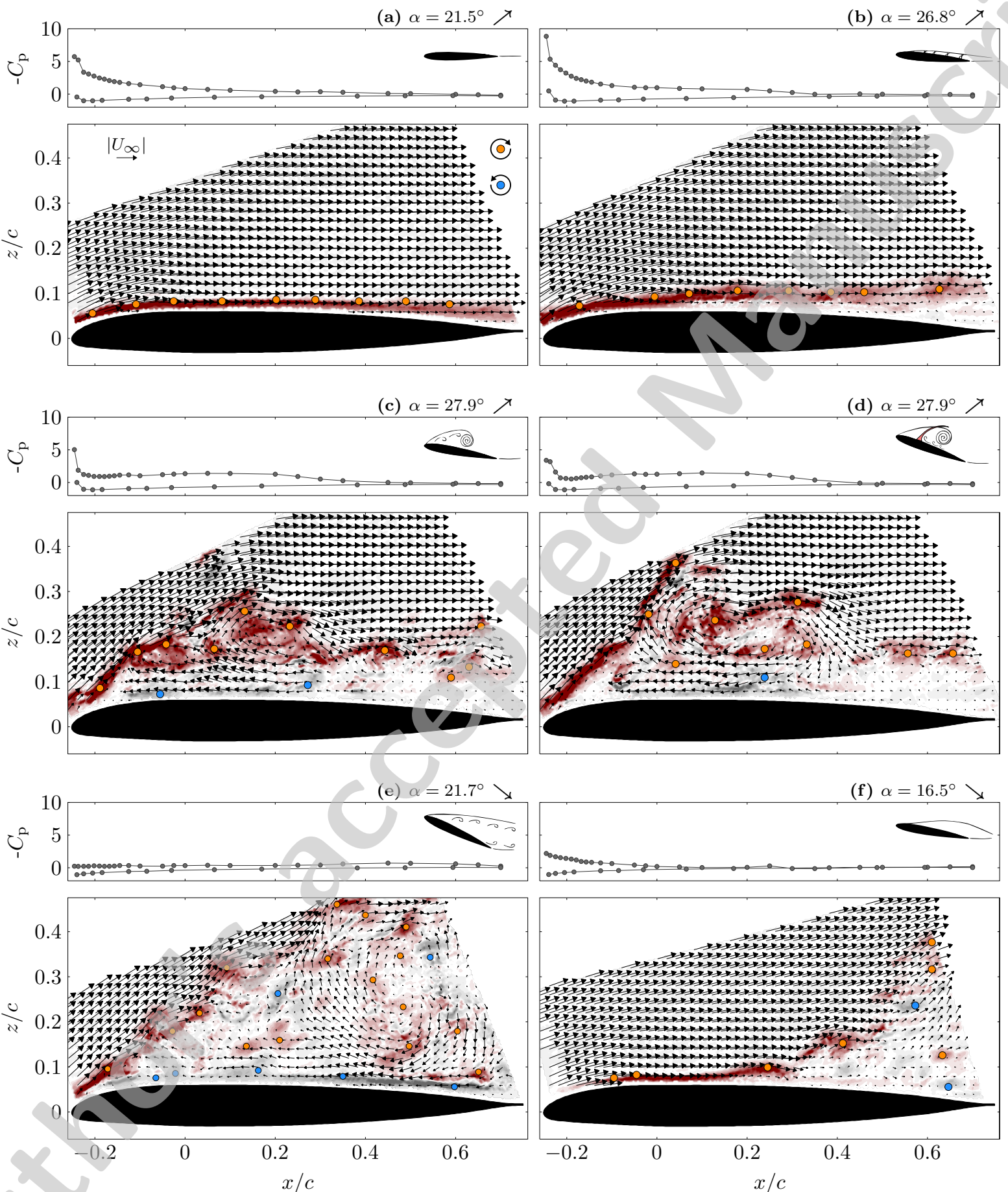

Fig. 3 Instantaneous flow fields with detected vortex cores: $(\bullet)$ clockwise and $(\bullet)$ anticlockwise rotation; and the respective surface pressure distribution for the different stall development stages within a single oscillation $\left(\alpha_{0}=20^{\circ}, \alpha_{1}=8^{\circ}\right.$, and $\left.k=0.10\right)$. Only every sixth velocity vector is shown for the sake of visibility and the dimensionless out-of-plane component of the vorticity is colour-coded. 
(a)

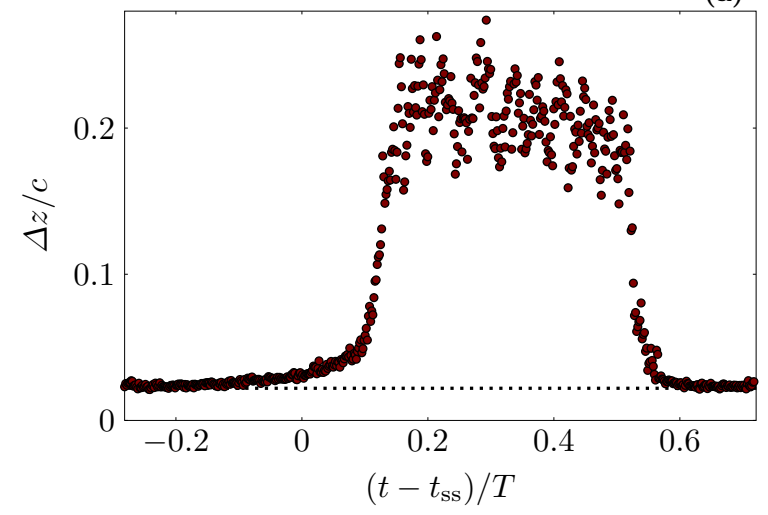

(b)

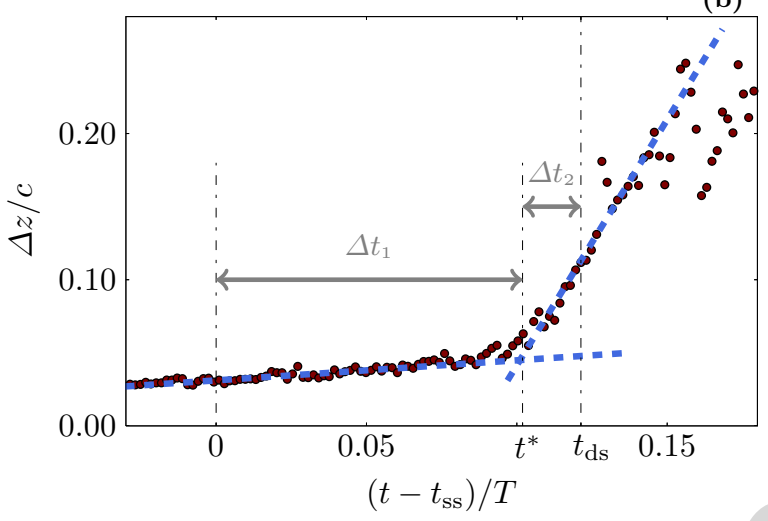

Fig. 5 Temporal evolution of the average shear layer height in terms of $\Delta z$ over one oscillation period $T$ (a), with a detailed view (b) including the corresponding linear fits to the data. The dotted line in (a) indicates the noise level due to experimental limitations. The time axis is shifted with respect to the passage of the static stall angle during upstroke $t_{\mathrm{ss}}$. The onset of dynamic stall is indicated by $t_{\mathrm{ds}}\left(\alpha_{0}=20^{\circ}, \alpha_{1}=8^{\circ}, k=0.05\right)$.

normal distance of the individual vortices $v_{\mathrm{i}}$ to the airfoil's upper surface as illustrated in figure 4 .

A representative temporal evolution of $\Delta z$ for one oscillation period $T$, which starts at minimum incidence, is depicted in figure 5 for $\alpha_{0}=20^{\circ}, \alpha_{1}=8^{\circ}$, and $k=0.05$. For small angles of attack, vorticity is a priori confined to the thin boundary layer and is convected downstream along the airfoil's contour. The convection dominated vorticity transport limits the boundary layer growth. For a fully attached flow $\Delta z$ is expected to be constant and of the order of the boundary layer thickness. Due to experimental limitations valid velocity information was only available down to approximately $2 \mathrm{~mm}$ above the airfoil's surface. With increasing angle of attack, $\Delta z$ increases until the flow is massively separated. Near the end of the cycle, $\Delta z$ decreases to its initial unstalled value, indicating reattachment of the flow.

\subsection{Two-stage Stall Development}

The most interesting behaviour of $\Delta z$ is observed during the stall development stage (figure 5). The stall development stage was defined earlier as the part of the DS life cycle where the angle of attack is between the static and dynamic stall onset angle. The static stall onset angle is determined from the static airload data as $a_{\mathrm{ss}}=21.4^{\circ}$. The time at which the static stall angle is exceeded in the DS cycle is denoted by $t_{\mathrm{ss}}$. The dynamic stall onset angle is specified directly from the measured velocity field based on a characteristic mode of the proper orthogonal decomposition (POD). More specifically, it is determined as the angle of attack for which the time development coefficient of the POD mode representing the large scale DS vortex reaches an extremum. This corresponds directly to the separation of the DS vortex (Mulleners and Raffel 2012). Dynamic stall onset is indicated by $a_{\mathrm{ds}}$ and $t_{\mathrm{ds}}$, respectively referring to the angle of attack and the time instant.

The evolution of $\Delta z$ during the stall development stage exhibits a two-stage linear increase with a distinctly different growth rate. The explanation for the difference and the sudden change in growth rate is provided based on close examination of the flow topology and vortex distribution during the two sub-stages (figure 6).

During the first part of stall development, which is characterised by the slow linear increase of $\Delta z$, the recirculation region grows steadily in chord-normal direction. Small-scale co-rotating vortices emerge in the shear layer due to a primary instability. These shear layer vortices are regularly spaced and all rotating clockwisely. They are convected downstream by the external flow and do not merge or overtake each other during this first sub-stage (figure 6(a)). By reference to standard mixing layer notations (Corcos and Sherman 1984), the initial part of stall development is referred to as the primary instability stage.

During the later part of stall development, which is characterised by a stronger, but still linear, increase of $\Delta z$, the viscous interactions between the small shear layer vortices increase. They merge as a result of a secondary instability leading to shear layer roll-up and the formation of the primary DS vortex. Therefore, this part of stall development is referred to as the vortex formation stage.

While the chord-normal distance of the shear layer smoothly increases during the primary instability stage due to the growth of the recirculation region, $\Delta z$ mainly increases due to chord-normal excursions and coalescence of the shear layer vortices during the vortex formation stage. When vortex pairs coalesce, the strength 


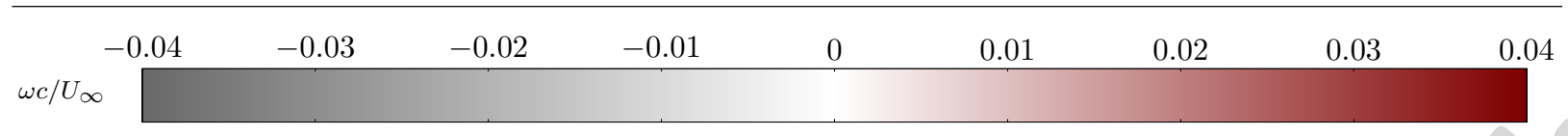

(a) $\alpha=25.5^{\circ} \nearrow$

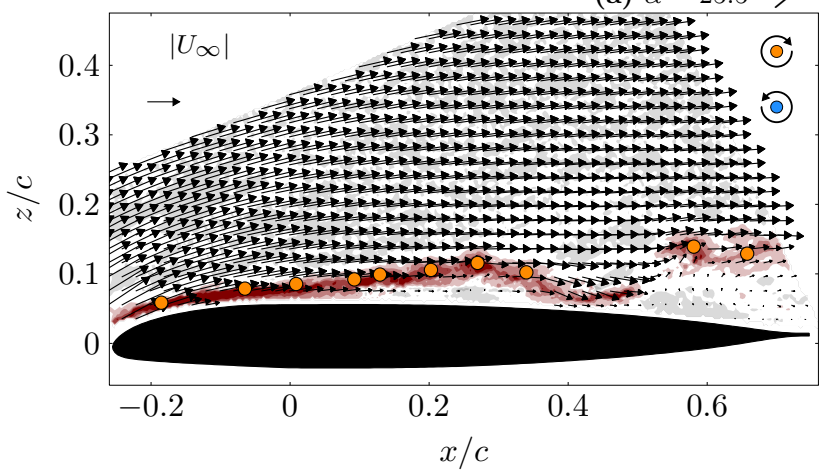

(b) $\alpha=26.4^{\circ}$

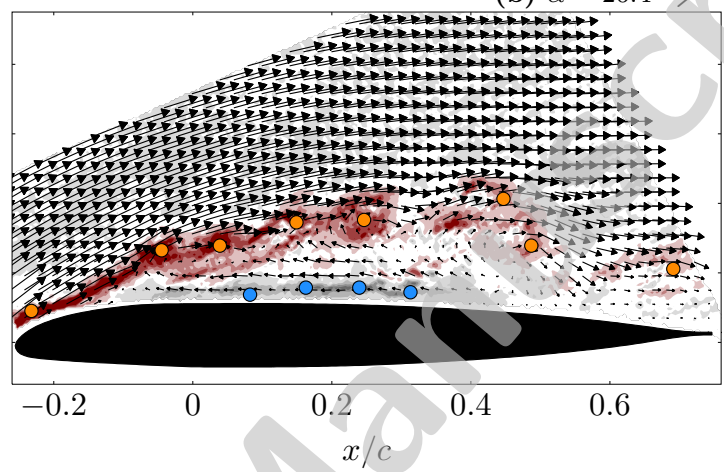

Fig. 6 Difference in flow topology and vortex distribution (clockwise $(\bullet)$ and counter-clockwise $(\bullet)$ rotating vortices) between the primary instability (a) and the vortex formation stage (b) $\left(\alpha_{0}=20^{\circ}, \alpha_{1}=8^{\circ}, k=0.050\right)$.

of the individual vortices and the spacing between them grows binomially doubling the time lag between subsequent coalescences. The growth of a free shear layer is therefore, on average, linear in time (Corcos and Sherman 1984) and the same is true for the increase of the mean height $\Delta z$ during the vortex formation stage (figure 5). The rate of increase of $\Delta z$ during this stage thus provides a measure for the rate of growth of the dynamic stall vortex.

In addition, the increased circulation around merged vortex pairs enforces stronger interactions between the viscous shear layer and the reversed flow generating counter-rotating vortices near the airfoil's surface (figure $6(\mathrm{~b})$ ). These anti-clockwise rotating vortices trigger the onset of dynamic stall as they move towards the leading edge and promote vortex induced separation with increased angle of attack (Mulleners and Raffel 2012).

\subsection{Transition of the Shear Layer Instability}

Based on the change in growth rate of the shear layer height and the different associated shear layer characteristics, stall development is subdivided here into the primary instability and the vortex formation stage. The transition between both regimes of stall development is specified in the Eulerian framework by the intersection of the two linear fitting curves that approximate the two-stage constant growth of $\Delta z$ in the individual regimes (figure $5(\mathrm{~b})$ ). The time instant and angle of attack at which the transition takes place is denoted by $t^{*}$ and $\alpha^{*}$, respectively.
A sequence of instantaneous negative FTLE fields around $t=t^{*}$ shows the evolution of the shear layer during transition from the primary instability stage, which is governed by inertial effects, and the vortex formation stage, which is governed by viscous effects. The ridges in the FTLE field represent material lines that segregate the inviscid free stream flow from the viscous flow within the shear layer. Bulging of the material lines indicate the engulfing process that characterises a secondary instability of the shear layer leading to shear layer roll-up. This process is comparable to the engulfing process during mixing layer roll-up described by Corcos and Sherman (1984) (figure $7(\mathrm{a})$ ).

The transition of the shear layer instability from a primary instability, generating small scale shear layer vortices, to a secondary instability, causing shear layer roll-up, induces an increase in the displacement rate of the shear layer and affects the aerodynamic performance of the airfoil. During the primary instability stage, the lift coefficient $C_{1}$ continuously increases and the first drop of $C_{1}$ coincides with the transition into the vortex formation stage. The lift overshoot with regard to static stall - which is the only desirable attribute of dynamic stall - is thus entirely generated during the primary instability stage.

The fundamental difference in shear layer behaviour has a direct impact on the lift coefficient. To improve current semi-empirical engineering models for the prediction of dynamic stall airloads, this is an important observation. In order to replace the empirical parameters in these models by physical relationships, comprehensive knowledge of the governing parameters and 

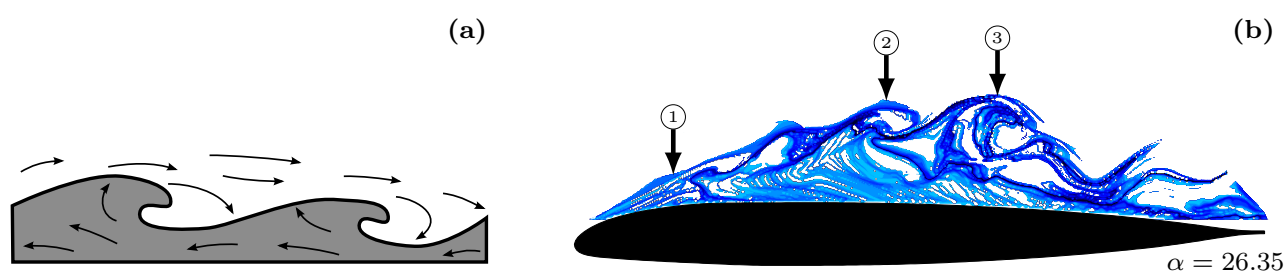

(b)
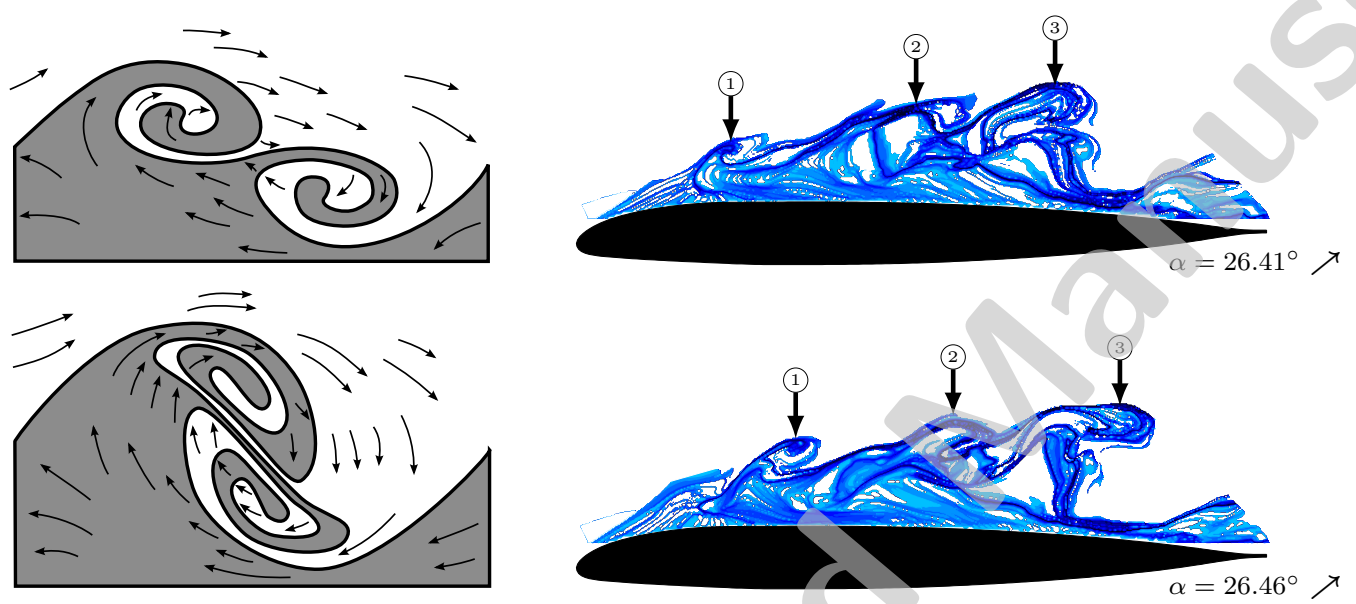

Fig. 7 Schematic representation of the engulfing process during mixing layer roll-up by Corcos and Sherman (1984) (a) and the development of a secondary instability of the shear layer visualised be the negative FTLE fields, extracted based on instantaneous velocity fields within a single oscillation $\left(\alpha_{0}=20^{\circ}, \alpha_{1}=8^{\circ}\right.$, and $\left.k=0.05\right)$, at consecutive time steps during transition from the primary instability into the vortex formation stage (b).

their effect on the stall delays attributed to the two substages of stall development is essential.

\subsection{Time Scales of Stall Development}

Stall delay refers to the time delay between static and dynamic stall onset. According to the splitting of the stall development stage, the overall stall delay is the sum of the time delays attributed to the primary instability and vortex formation stage denoted by $\Delta t_{1}$ and $\Delta t_{2}$, respectively (figure 5(b)). The separate contributions are specified as $\Delta t_{1}=t^{*}-t_{\mathrm{ss}}$ and $\Delta t_{2}=t_{\mathrm{ds}}-t^{*}$.

In order to be able to predict the stall delay for arbitrary dynamic stall oscillations in the furture, we need to determine in what pathway the airfoil dynamics affect the time delay contribution of both substages. For a sinusoidal oscillation, the airfoil's motion is described by the interrelated parameters mean incidence, amplitude, and oscillation frequency. These parameters can be combined into a single representative parameter, the instantaneous effective unsteadiness $\dot{\alpha}_{\text {ss }}$. The instantaneous effective unsteadiness is defined as the rate of change of the angle of attack when the static stall angle is exceeded. It successfully describes the influence of the unsteadiness of the pitching motion with respect to the dynamic stall process (Mulleners and Raffel 2012). In particular, a hastened onset of dynamic stall was observed previously with increasing effective unsteadiness.

Considering now the individual stall delays $\Delta t_{1}$ and $\Delta t_{2}$ as a function of the normalised effective unsteadiness $\dot{\alpha}_{\mathrm{ss}} c / U_{\infty}$, additional information is obtained (figure $9(\mathrm{a}))$. Firstly, the delay due to the primary insta-

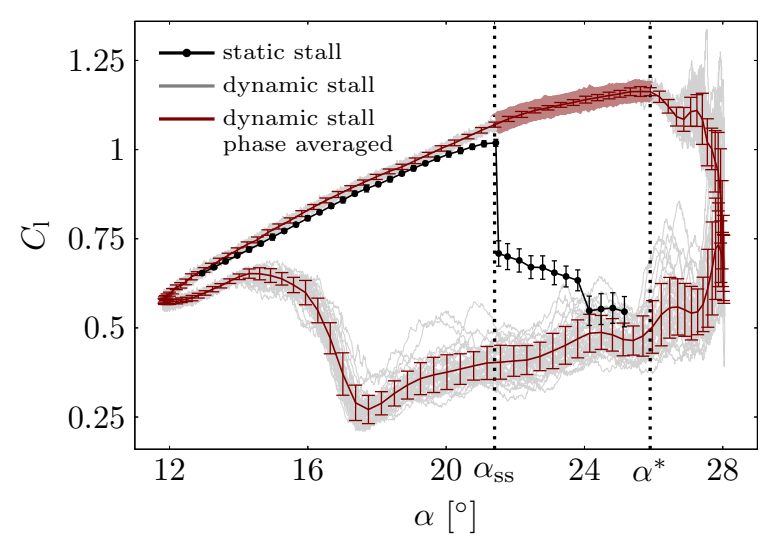

Fig. 8 Lift coefficient as a function of angle of attack for static and dynamic stall. The coloured segment corresponds to the primary instability stage $\left(\alpha_{0}=20^{\circ}, \alpha_{1}=8^{\circ}, k=0.05\right)$. 
(a)

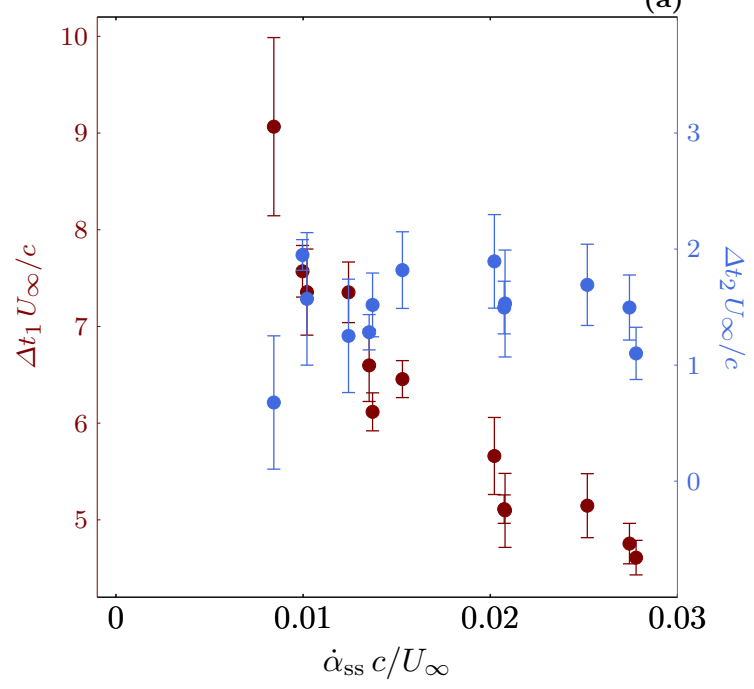

(b)

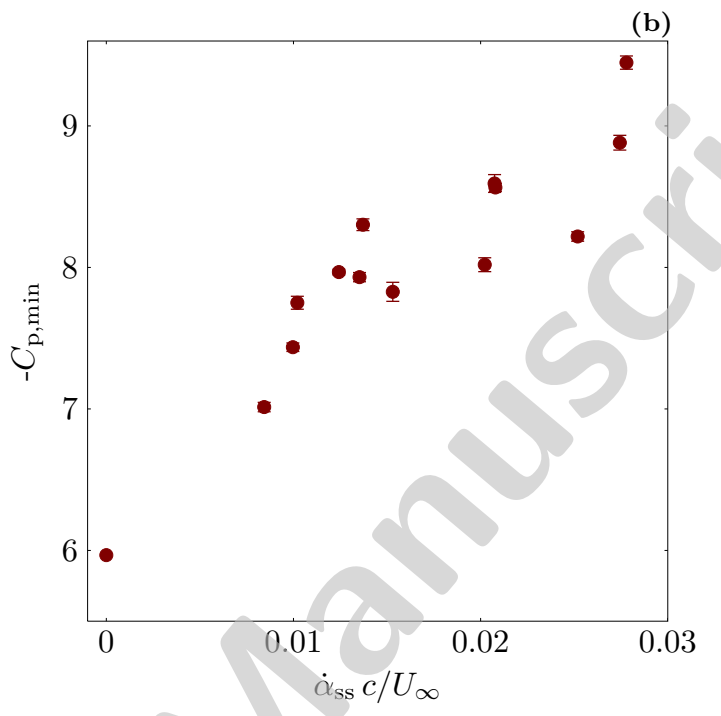

Fig. 9 Influence of the instantaneous effective unsteadiness on the dynamic stall delays $\Delta t_{1}$ and $\Delta t_{2}$ attributed to the primary instability stage and the vortex formation stage, respectively (a); the minimum surface pressure level $C_{\mathrm{p}, \text { min }}(\mathrm{b})$. Error bars indicate the standard deviations.

bility stage is at least double the delay time due to the vortex formation stage. Secondly, $\Delta t_{2}$ is approximately constant for all pitching motions considered here. There is no significant influence of the airfoil's motion on the vortex formation process because the shear layer rollup is mainly governed by viscous interactions between the shear layer vortices. Finally, $\Delta t_{1}$ clearly decreases with increasing effective unsteadiness. When the pitch rate increases, a larger amount of vorticity is generated and accumulates in the shear layer (Acharya and Metwally 1992). The more vorticity accumulates in the shear layer, the faster a secondary shear layer instability sets in, hence the shorter the primary instability stage which ends with the transition of the shear layer at $t^{*}$. Evidence that vorticity in the shear layer increases with increasing effective unsteadiness is provided in figure 9 (b) because increased vorticity in the shear layer leads to a stronger acceleration of the flow or an increased suction peak at the leading edge (Lorber and Carta 1988; Visbal and Shang 1989). As a consequence, the minimum suction peak at dynamic stall must always be larger than at static stall, which is true for the present experiments. In figure $9(\mathrm{~b})$, the static reference case fits well within the trend revealed by the dynamic test conditions.

\section{Conclusion and Future Work}

The dynamic stall development on a pitching airfoil was discussed based on a combination of time-resolved velocity field information and coherent structure analysis.
Stall development describes the period of time prior to stall onset and covers the emergence and subsequent development of a shear layer at the interface between recirculating and free stream flow. The shear layer is the prominent feature during stall development. Based on different shear layer behaviour, the stall development stage was subdivided into a primary instability stage followed by a vortex formation stage. The primary instability stage essentially covers the growth of the recirculation region and the corresponding initial development and primary instability of the shear layer. The transition from a primary into a secondary shear layer instability leads to a sudden increase in the rate of displacement of the shear layer and the first drop of the lift coefficient. The transition of shear layer instability marks the beginning of the second part of stall development or vortex formation stage. The vortex formation stage is characterised by the roll-up of the shear layer into the large-scale DS vortex which grows locally and temporally until separating as a result of vortex induced separation. The scenario was identified as wake mode.

The overall stall delay is the sum of the time delays attributed to the primary instability and vortex formation stage, where the former is at least double as long as the latter. The delay attributed to the primary instability stage decreases with increasing unsteadiness and is correlated with the minimum surface pressure level. The larger the instantaneous effective unsteadiness, the more vorticity accumulates in the shear layer, leading to increased suction peaks and promotion of the onset of shear layer roll-up. The relatively small delay 
attributed to the vortex formation stage is governed by viscous flow interactions and virtually unaffected by variations in the airfoil's pitching motion.

The combination of multiple vortex identification procedures that are different in nature provides a more detailed and more comprehensive insight into unsteady vortex flows than conventional methods are capable of. Future work will focus on: the dynamics of static stall development, three-dimensional aspects of dynamic stall as well as dynamic stall in rotating environments.

Acknowledgements This work has been part of the DLR and ONERA joint project: Advanced Simulation and Control of Dynamic Stall (SIMCOS). The authors thank H. Mai, T. Büte, J. Nuhn and A. Henning for their assistance during the wind tunnel measurements and K. Kindler for comments and discussion.

\section{References}

Acharya M, Metwally MH (1992) Unsteady Pressure Field and Vorticity Production over a Pitching Airfoil. AIAA Journal 30(2):403-411

Carr L, McAlister K, McCroskey W (1977) Analysis of the Development of Dynamic Stall Based on Oscillating Airfoil Experiments. Tech. rep., NASA

Carr LW (1988) Progress in Analysis and Prediction of Dynamic Stall. Journal of Aircraft 25(1):6-17

Corcos G, Sherman F (1984) The mixing layer: deterministic models of a turbulent flow. Part 1. Introduction and the two-dimensional flow. Journal of Fluid Mechanics 139:29-65, DOI 10.1017/S0022112084000252, URL http://www.journals.cambridge.org/abstract_S00221120840002

Degani A, Walker J, Smith F (1998) Unsteady separation pas moving surfaces. Journal of Fluid Mechanics 375:1-38

Doligalski T, Smith C (1994) Vortex interactions with walls. Annual review of fluid 26:573-616, URL http://www.annualreviews.org/doi/pdf/10.1146/annurev.fl.26.010194.003041

Garcia D (2010) A fast all-in-one method for automated post-processing of PIV data. Experiments in Fluids 50(5):1247-1259, DOI 10.1007/s00348-010-0985-y, URL http://www.springerlink.com/index/10.1007/s00348-0100985-y

Gharib M, Roshko A (1987) The effect of flow oscillations on cavity drag. Journal of Fluid Mechanics 177:501- 530

Haller G, Yuan G (2000) Lagrangian coherent structures and mixing in two-dimensional turbulence. Physica D: Nonlinear Phenomena 147(3-4):352370, DOI 10.1016/S0167-2789(00)00142-1, URL http://linkinghub.elsevier.com/retrieve/pii/S0167278900001421

Ho CM (1986) An alternative look at the unsteady separation phenomenon. In: Recent advances in aerodynamics, Springer, chap An alterna, pp 165-178

Hudy LM, Naguib A, Humphreys WM (2007) Stochastic estimation of a separated-flow field using wallpressure-array measurements. Physics of Fluids 19(2):024,103-1-18, DOI 10.1063/1.2472507, URL http://link.aip.org/link/PHFLE6/v19/i2/p024103/s1\&Agg=doi

Lekien FF, Coulliette C, Mariano A, Ryan E, Shay L, Haller G, Marsden J (2005) Pollution release tied to invariant manifolds: A case study for the coast of Floridalease tied to invariant manifolds: A case study for the coast of Florida. Physica D: Nonlinear Phenomena 210:1-20
Lorber P, Carta F (1988) Airfoil Dynamic Stall at Constant Pitch Rate and High Reynolds Number. Journal of Aircraft 25(6):548-556

McAlister K, Carr L, McCroskey W (1978) Dynamic Stall Experiments on the NACA 0012 Airfoil. Tech. rep., NASA

McCroskey WJ (1981) The Phenomenon of Dynamic Stall. Tech. rep., NASA

Michard M, Graftieaux L, Lollini L, Grosjean N (1997) Identification of Vortical Structures by a non Local Criterion Application to PIV Measurements and DNS-LES Results of Turbulent Rotating Flows. In: Proceedings of the 11th Conference on Turbulent Shear Flows

Mulleners K, Raffel M (2012) The onset of dynamic stall revisited. Experiments in Fluids 52(3):779-793, DOI 10.1007/s00348-011-1118-y, URL http://www.springerlink.com/index/10.1007/s00348-011$1118-\mathrm{y}$

Obabko aV, Cassel KW (2002) Detachment of the dynamic-stall vortex above a moving surface. AIAA Journal 40(9):1811-1822, DOI 10.2514/3.15264, URL http://arc.aiaa.org/doi/abs/10.2514/3.15264

Raffel M, Willert CE, Wereley ST, Kompenhans J (2007) Particle Image Velocimetry: A Practical Guide. Experimental Fluid Mechanics Series, Springer, URL http://books.google,de/books?id=WH5w2TvK2bQC

Reynolds W, Carr L (1985) Review of Unsteady, Driven, Separated Flows. Tech. rep., AIAA

Shadden SC, Lekien F, Marsden JE (2005) Definition and properties of Lagrangian coherent structures from finitetime Lyapunov exponents in two-dimensional aperiodic flows. Physica D: Nonlinear Phenomena 212(34):271-304, DOI 10.1016/j.physd.2005.10.007, URL http://linkinghub.elsevier.com/retrieve/pii/S0167278905004446

Shih C, Lourenco L, Van Dommelen L, Krothapalli A (1992) Unsteady Flow Past an Airfoil Pitching at a Constant Rate. AIAA Journal 30(5):1153-1161

Visbal MR, Shang JS (1989) Investigation of the Flow Structure Around a Rapidly Pitching Airfoil. AIAA Journal 27(8):1044-1051 